\title{
Síndrome de Fournier: Análise dos Fatores de Mortalidade
}

\author{
Analysis of Mortality in Fournier's Gangrene
}

\author{
PAULO DE AZEREDO PASSOS CANDELÁRIA ${ }^{1}$; WILMAR ARTUR KLUG ${ }^{2}$; PERETZ CAPELHUCHNIK ${ }^{3}$; \\ CHIA BIN FANG ${ }^{4}$
}

\begin{abstract}
1. Professor Instrutor da Faculdade de Ciências Médicas da Santa Casa de São Paulo - Médico Assistente do Serviço de Emergência da Irmandade da Santa Casa de Misericórdia de São Paulo; ${ }^{2}$ Professor Titular da Faculdade de Ciências Médicas da Santa Casa de São Paulo - Médico Chefe de Disciplina de Coloproctologia; ${ }^{3}$ Professor Titular da Faculdade de Ciências Médicas da Santa Casa de São Paulo - Médico Chefe de Clínica; ${ }^{4}$ Professor Adjunto do Departamento de Cirurgia da Faculdade de Ciências Médicas da Santa Casa de São Paulo - Médico Primeiro Assistente Departamento de Cirurgia da Irmandade da Santa Casa de São Paulo.
\end{abstract}

\begin{abstract}
CANDELARIA PAP; KLUG WA; CAPELHUCHNIK P; FANG CB. Síndrome de Fournier: Análise dos Fatores de Mortalidade. Rev bras Coloproct, 2009;29(2): 197-202.
\end{abstract}

RESUMO: A fascite necrótica perineal (Síndrome de Fournier) é infecção grave dos tecidos moles, de etiologia não totalmente esclarecida, porém associada a procedimentos urológicos, proctológicos ou ginecológicos, além de diabetes melito, alcoolismo, desnutrição grave e outros estados de imunodepressão. Trata-se de situação grave, exigindo antibioticoterapia de amplo espectro, desbridamentos cirúrgicos e por vezes derivação do trânsito fecal e/ou urinário. OBJETIVO: Avaliar os fatores relacionados com mortalidade. CASUÍSTICA: Foram avaliados retrospectivamente 43 doentes (39 masculinos) com média de idade de 54,8 anos, no período de 1998 a 2005, na Santa Casa de São Paulo. MÉTODO: foram analisados sexo, idade, sinais e sintomas, tempo de evolução e de internação, doenças associadas, sepse, broncopneumonia, exames laboratoriais, área comprometida, cirurgias realizadas, índice fisiológico Apache II e causa de óbito. RESULTADOS: Sobreviveram 33 doentes e houve 10 (23,2\%) óbitos. A letalidade relacionou-se a pacientes mais velhos, tempo de evolução longo, internações curtas, índice fisiológico Apache II elevado, sepse e broncopneumonia . Não houve relação com sexo, diabetes melito, desnutrição, culturas e antibioticoterapia, local de início, extensão da necrose, número de desbridamentos, bem como com colostomias e cistostomias. CONCLUSÃO: a letalidade relaciona-se à disseminação da infecção e retardo do tratamento. Não é relacionada com diabete nem cirurgias como colostomia ou cistostomia.

Descritores: Síndrome de Fournier, gangrena, infecção, letalidade.

\section{INTRODUÇÃO}

A Síndrome de Fournier é uma fascite necrótica que acomete os tecidos moles da região perineal. Pode estar relacionada a focos iniciais no trato gênito-urinário ou na região anal ${ }^{1}$, injeções de drogas ${ }^{2}$, ferimentos do períneo e abscessos perianais ${ }^{3}$. Pode ser também observada após procedimentos urológicos, ginecológicos e anais, mesmo em recém nascidos ${ }^{4}$. Há causas raras, como introdução de próteses penianas ${ }^{5}$. Ocorrem doenças associadas, como diabetes melito que pode chegar até $60 \%$ dos casos ${ }^{6}$. Além disso, idade avançada, hospitalização prolongada, carcinoma e alcoolismo são mencionados ${ }^{7}$, além de corticoterapia, desnutrição, radioterapia, quimioterapia, imunossupressão, defeitos sensoriais, falência renal, hemodiálise, vasculite, cirrose, lúpus e SIDA ${ }^{8}$.

O tratamento é cirúrgico de urgência, com desbridamento do tecido desvitalizado. Se o processo progride, é necessário reoperar. A letalidade varia, e

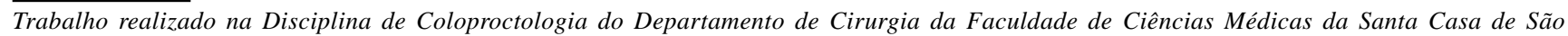
Paulo - SP - Brasil. 
mesmo após cirurgia agressiva, as taxas de mortalidade são em parte decorrentes da agressividade da infecção e de doenças associadas 9,10 .

Em função da baixa frequência, diversidade terapêutica e carência de parâmetros definidos para caracterizar fatores associados e extensão anatômica do processo infeccioso, os fatores de letalidade não estão claros, havendo muitas divergências ${ }^{10}$.

O objetivo do trabalho foi avaliar os fatores relacionados à mortalidade em pacientes atendidos na Santa Casa de São Paulo, para estabelecer a importância da extensão da infecção, doenças associadas e o tratamento operatório, visando especificar quais se relacionam com mortalidade.

\section{MÉTODO}

Analisamos 43 doentes internados desde 1999 até 2006, após aprovação da pesquisa pelo Comitê de Ética em Pesquisa da S. Casa de S. Paulo. Trata-se de estudo retrospectivo com base em análise de prontuários do hospital. Foram 39 pacientes do sexo masculino e quatro do sexo feminino, com idades variando entre 23 a 86 anos, média de 54,8. O tempo de evolução foi entre três e 30 dias, média de 10,9. O tempo de internação foi de dois a 93 dias, média de 26,4.

Para estudar o seu impacto na mortalidade analisamos estatisticamente todas as informações abaixo relacionadas como: gênero, idade, sintomas, tempo de evolução e de internação e doenças associadas, bem como parâmetros hemodinâmicos e frequência respiratória. Foram testados exames laboratoriais como pressão parcial de oxigênio, pH arterial, creatinina, sódio e potássio séricos, hematócrito e leucometria, e também resultados de culturas e antibiograma. Analisamos as complicações, tratamentos e índices de mortalidade sendo os pacientes classificados pelo método APACHE II. Ao avaliar objetivamente o local de início e extensão da necrose, dividimos o tamanho da região comprometida; em um grupo menor e outro maior que cinco centímetros, sendo os limites dados pela divisão do períneo em dois triângulos: urogenital e anal.

Houve pacientes submetidos inicialmente à drenagem de abscesso com anestesia local; e por agravamento seguiu-se desbridamento repetido. Todos foram submetidos à excisão da área de necrose, estendendo a ressecção até haver sangramento das bordas. Em alguns realizou-se colostomia por extensa área de necrose perianal e cistostomia, se dificultada a mic- ção. O número e o tipo de cirurgias também foram estatisticamente analisados. Realizaram-se culturas das secreções e antibiograma. A terapia antibiótica variou consoante as equipes que inicialmente conduziram os tratamentos. O esquema mais utilizado foi associação ampicilina, cloranfenicol e amicacina. Para análise estatística os doentes foram divididos em sobreviventes e óbitos e para quantificação das variáveis foi aplicado o teste de $\div^{2}$, em tabelas de dupla entrada. Havendo significância estatística foi usada a correlação de Pearson entre as variáveis selecionadas. $\mathrm{O}$ nível de significância adotado foi de 0,05 . Através de método estatístico com inclusão de análise descritiva dos dois grupos e a aplicação do teste t de Student, procuramos estabelecer um valor de risco para o índice fisiológico APACHE II.

\section{RESULTADOS}

Todos os fatores analisados foram relacionados à mortalidade para avaliar a existência ou não de diferença significante. Faleceram nove pacientes do sexo masculino e uma do feminino, não havendo diferença de gênero entre óbitos e sobrevivência $(p=0,593)$. Com relação à idade, os pacientes que sobreviveram foram mais jovens $(\mathrm{p}<0,001)$. Os aspectos clínicos, como queixas de dor, prurido, febre e edema ao exame, não foram diferentes na distribuição amostral entre os sobreviventes e óbitos ( $\mathrm{p}>0,05)$. Contudo, o tempo de evolução da doença foi maior entre os óbitos $(\mathrm{p}=0,009)$. A média em dias de internação entre os que sobreviveram (30,9 dias) foi muito maior que dos óbitos (11,7 dias), justificada pelo controle da infecção e cirurgias repetidas.

Houve anotação da presença de doenças associadas, presentes em $32(74,4 \%)$ (Tabela 1).

Selecionamos as principais doenças para verificar sua relação com a mortalidade, através da correlação de Pearson. A correlação não foi positiva com desnutrição, apesar de ser a co-morbilidade mais comum $(\mathrm{p}=0,09)$. Da mesma forma, a presença de diabetes melito não teve relação com a mortalidade $(\mathrm{p}=0,98)$. A presença de sepse, porém, diagnosticada pelos parâmetros habitualmente considerados, foi claramente fator determinante de óbito ( $p<0,001)$. Infecção do trato urinário não foi significativa, presente em sete dos sobreviventes e dois dos que foram a óbito $(\mathrm{p}=0,93)$. Ao contrário, infecção pulmonar foi decisiva, presente em seis dos mor- 
tos e só em um sobrevivente $(\mathrm{p}<0,001)$. Leucocitose $(\mathrm{p}=0,34)$ não teve significado e acidose $(\mathrm{p}=0,001)$ parece relacionada ao quadro geral de sepse responsável pelas mortes.

Com respeito às bactérias responsáveis pela infecção inicial, em 33 sobreviventes foram solicitadas culturas, ou da secreção inicial, ou durante a evolução hospitalar. Vinte e cinco resultaram positivas e duas negativas. Entre as positivas houve germes gram positivos ou negativos, mas em $15(60 \%)$ a flora foi mista. Entre os cocos gram + ressaltaram os S. aureus (10) além de Streptococcus do grupo D (3) e Enterococcus (2). Entre os cocos gram - observamos E. coli (9), $K$. pneumonie (4), P. aeruginosa (3). Anaeróbios como Bacteróides $s p$. foram identificados em seis culturas mistas. Em um paciente ocorreu Candida $s p$. Antibiograma foi realizado em 21 casos, e em 14 o esquema inicial teve sensibilidade adequada, o que não ocorreu em sete. No grupo óbito, a relação de germes seguiu o mesmo padrão. Dez culturas foram solicitadas. Em uma delas a infecção resultou de contaminação múltipla. Não observamos diferença entre o pa- drão das culturas entre sobreviventes e falecidos. Das dez culturas os antibiogramas indicaram sensibilidade adequada em dois casos ao esquema originalmente prescrito. Os esquemas antibióticos iniciais foram posteriormente alterados em decorrência da orientação dada pelo antibiograma.

Em relação à área do comprometimento inicial e sua extensão, os resultados indicaram que a letalidade foi independente do local do início, como também do tamanho da lesão inicial, maior ou menor que $5 \mathrm{~cm}$ $(\mathrm{p}=0,19)$.

Os procedimentos cirúrgicos iniciais e subsequentes estão relacionados na Tabela 2. A correlação de Pearson não demonstrou diferença entre eles. O mesmo resultado foi observado ao comparar os pacientes que sofreram um ou múltiplos desbridamentos $(\mathrm{p}=0,32)$.

Cistostomias, realizadas em oito (um óbito) e colostomias, realizadas em 19 (seis óbitos), não foram estatisticamente significantes por não terem sido fator de proteção contra óbito ( $\mathrm{p}=0,43$ e 0,06 , respectivamente).

Tabela 1 - Distribuição amostral das doenças associadas listadas de acordo com a frequência e sua significância estatística.

\begin{tabular}{lccc}
\hline Doenças associadas & $\mathbf{n}^{\circ}$ & $\mathbf{\%}$ & $\boldsymbol{\rho}$ \\
\hline Desnutrição & 16 & 37,2 & 0,09 \\
Diabetes melito & 13 & 30,2 & 0,98 \\
Sepse & 10 & 23,2 & $<0,001$ \\
Infecção do trato urinário & 9 & 20,9 & 0,93 \\
Broncopneumonia & 7 & 16,2 & $<0,001$ \\
Hipertensão arterial sistêmica & 6 & 13,9 & $>0,05$ \\
Insuficiência cardíaca congestiva & 4 & 9,3 & $>0,05$ \\
Etilismo & 4 & 9,3 & $>0,05$ \\
Arritmia cardíaca & 3 & 6,9 & $>0,05$ \\
Síndrome da imunodeficiência adquirida & 3 & 6,9 & $>0,05$ \\
\hline
\end{tabular}

Tabela 2 - Distribuição amostral de pacientes sobreviventes ou não em relação ao procedimento cirúrgico.

\begin{tabular}{lccc}
\hline Procedimento cirúrgico & Sobreviventes & Óbitos & Total \\
\hline Drenagem no Pronto Socorro & 7 & 1 & 8 \\
Desbridamento no Pronto Socorro & 1 & 0 & 1 \\
Drenagem no Centro Cirúrgico & 9 & 0 & 9 \\
Desbridamento no Centro Cirúrgico. & $33(\mathrm{p}=0,32)$ & 9 & 42 \\
\hline
\end{tabular}


A medição do Índice Fisiológico APACHE II, contudo, foi preciso para indicar o risco de óbito, cujos valores maiores que 19 são claramente preditivos $(\mathrm{p}=0,001)$.

A causa dos óbitos, bem como a extensão das lesões e as complicações estão relacionadas na Tabela 3 .

\section{DISCUSSÃO}

Há referências à doença desde a antiguidade e a mortalidade média referida de $22 \%$ não foi reduzida até hoje ${ }^{11}$. Em relação à idade, parece claro que paciente jovem tem melhor prognóstico ${ }^{12}$. Não houve óbitos em pacientes mais jovens que 50 anos. O tempo de internação é extremamente variável. No grupo dos que foram a óbito o tempo de internação foi menor, pois os sobreviventes são tratados com maior intensidade por maior tempo. Também varia o tempo de evolução da doença antes da internação, que entre os sobreviventes foi menor, confirmando o conceito de que o retardo no tratamento é seguido por mais letalidade.

Em relação aos achados laboratoriais, verificou-se que as alterações do hematócrito, uréia, cálcio, albumina, fosfatase alcalina e colesterol são significantes ${ }^{13}$. Em nosso material, acidose foi fator de letalidade, mas não leucocitose.

Com relação à etiologia há grande variedade de situações relacionadas ${ }^{11}$. Nesta casuística foi possível identificá-la em 95,4\%. Tiveram origem no triângulo urogenital $37,2 \%$ dos casos e no triângulo anal $34,8 \%$, e não houve diferença na mortalidade o local da lesão inicial. Com relação à bacteriologia, observamos que a flora é mista, embora tenha-se dado importância ao choque estreptocócico ${ }^{9,14}$. Verificamos $60 \%$ de culturas microbianas em pacientes sobreviventes, e $20 \%$ nos que foram a óbito. As bactérias mais isoladas foram Staphylococcus aureus e Escherichia coli, e a presença de anaeróbios foi menor.

Diabetes complica a evolução de 40 a $60 \%$ dos pacientes ${ }^{1,3,15}$, e é imputado como principal responsável pelos maus resultados do tratamento. No entanto, há estudos que contrariam esta afirmação ${ }^{13}$. Observamos que os pacientes com história de diabetes melito são vulneráveis às infecções, mas a distribuição de diabéticos entre os grupo de sobreviventes ou não foi a mesma, não existindo correlação. $\mathrm{O}$ alcoolismo também não foi fator relevante. A associação com HIV deve ser considerada, pois três eram portadores $(6,9 \%)$ e um foi a óbito. Estados debilitantes podem contribuir com impacto negativo na sobrevivência ${ }^{13}$. Observamos que desnutrição foi o fator associado mais comum (37,2\%), não havendo correlação estatística.

Em relação à extensão da lesão, cita-se que maior necrose eleva os índices de mortalidade ${ }^{1,12,15}$; porém observamos que a extensão média da área de superfície corpórea envolvida em pacientes que sobreviveram $(4,32 \%)$ e faleceram $(7,16 \%)$ não foi estatisticamente significante, sugerindo-se que a extensão da infecção não é fator preditivo ${ }^{13}$ para mortalidade.

Com relação ao tratamento clínico, diversos esquemas antibióticos são utilizados, mas não parecem interferir nos resultados. Na questão técnica cirúrgica, desbridamento cirúrgico precoce e agressivo parece fundamental. Observação cuidadosa é essencial para

Tabela 3 - Correlação entre a extensão da área comprometida e a causa de óbito.

\begin{tabular}{lll}
\hline Extensão & Complicação & Causa de óbito \\
\hline hipogástrio+períneo anterior & broncopneumonia & sepse \\
coxa+abdome & insuficiência respiratória & sepse \\
Pós-oper. Hérnia inguinal & & sepse \\
períneo + escroto & broncopneumonia & sepse \\
escroto & broncopneumonia & sepse \\
escroto+coxa E.+D & broncopneumonia & sepse \\
escroto+ períneo & broncopneumonia & sepse \\
escroto + escara sacral & & sepse \\
escroto + raiz da coxa D & broncopneumonia & sepse \\
escroto+ períneo+ reg.ingu. bil.+coxa D & & sepse \\
\hline
\end{tabular}


perceber progressão da necrose e indicar novo desbridamento ${ }^{3,6}$. Não é uniforme, nem se conhece o número de procedimentos necessários para evitar óbito 3,13. Em relação à colostomia há controvérsias ${ }^{5,12,15}$. Em nossa casuística foram realizadas em $44,2 \%$, e a análise não mostrou vantagem comparativa. Em relação à cistostomia também há controvérsias. Nos pacientes submetidos ao procedimento não houve benefício em relação aos demais.

Complicações sistêmicas sérias podem ocorrer: insuficiência renal e síndrome da angústia respiratória, insuficiência cardíaca, pneumonia, hemorragia cerebral, coagulopatia, acidose, extensão da gangrena ao tronco, disfunção hepática e abscessos disseminados. Nos pacientes que foram a êxito letal, sepse foi a complicação mais frequente e a letalidade esteve claramente relacionada à infecção sistêmica. Em seis foi identificada broncopneumonia e nestes sepse foi considerada a causa do óbito.
$\mathrm{Na}$ admissão dos pacientes o índice fisiológico APACHE II é método que pode ser usado como fator preditivo. O índice APACHE II revelou-se excelente indicador, pois quando ele ultrapassou 19 a progressão para óbito $(\mathrm{p}=0,001)$ foi inevitável.

Apesar dos esforços no tratamento do paciente, houve alta taxa de mortalidade $(23,2 \%)$, semelhante à média histórica, sendo denominadores comuns faixa etária superior a 50 anos, tempo de evolução prolongado, infecção respiratória e septicemia, acidose e índice elevado do APACHE II.

\section{CONCLUSÃO}

Concluímos que o óbito ocorreu em pacientes com doença prolongada, disseminação da infecção e comprometimento dos pulmões, o que pôde ser previsto pelo acompanhamento clínico, e antecipado pela evidência de septicemia.

ABSTRACT: BACKGROUND: Necrotizing fasciitis is a severe infection of soft tissues, and when it affects the perineum it is called Fournier's gangrene.Some controversy exists over the origin of the disease, but all studies identified correlations between infection and proctologic, urologic, gynecologic origin procedures or trauma. In addition, many other causes are commonly associated, such as diabetes mellitus, abuse of ethanol, malnutrition and immunodepression. It is a severe situation, and diagnosis and surgical therapeutics are emergency procedures, and antibiotics and operations are usually required. OBJECTIVE: Evaluation of conditions related to the causes of death. METHOD: Since 1998 to 2005 in Santa Casa de São Paulo Hospital has 43 Fournier's gangrene patients (39 male) age averege 54,8 years and this study analysed their gender, symptoms, evolution of the disease, associated diseases, medical and surgical procedures, sepsis, lung infection, extension of infection, number of debridements, Apache II physiologic index and causes of death. RESULTS: The survivors was 33 pacients and overall mortality of 10 subjects $(\mathbf{2 3 . 2 \%})$, was related to age, delay of treatment, Apache II index and occurence of sepsis and pneumonia. No correlations were found with gender, diabetes mellitus, weight loss, bacteriology, place of begining and extension of lesions, number of debridments or colostomy and cistostomy. CONCLUSIONS: Mortality is related to treatment delay and sepsis. There is no statistical evidence of any relation with diabetes, colostomy or cistostomy.

Keywords: Fournier's gangrene, gangrene, infection, mortality.

\section{REFERÊNCIAS}

1. Paty R, Smith AD. Gangrene and Fourniers gangrene . Urol Clin N Amer 1992; 19: 149-160.

2. Dunbar NM, Harruf RC. Necrotizing fasciitis: manifestations, microbiology, and connection with black tar heroin. J Forens Sci 2007; 52(4): 920-923.

3. Steinman R, Utiyama EM, Maximiniano LF, Bevilacqua RG, Birolini D. Síndrome de Fournier: avaliação e tratamento inicial. Rev Assoc Med Bras 1992; 38: 209-213.

4. Christensen RD, Pysher TJ, Christensen SS. Case report.: perianal necrotizing fasciitis in a near-term neonate. J Perinatol 2007; 27(6): 390-391.
5. Hollabough Jr RS, Dmochowski RR, Hickerson WL, Cox CE. Fourniers gangrene: therapeutic impact of hyperbaric oxigen. Plast Reconst Surg 1998; 101(1): 94-100.

6. Thambi Dorai CR, Kandasami P. Fourniers gangrene: its aetilogy and management. Aust N Z J Surg 1991; 61: 3710372.

7. Theiss M, Hofmockel G, Frohmüller HG. Fourniers gangrene in a patient with erectile dysfunction following use of mechanical erection aid device. J Urol 1995; 153: 1921 1922.

8. Murphy M, Buckley M, Corr J, Vinayagamoorthy S, Grainger R, Mulcahy FM. Fourniers gangrene of scrotum in a patient with Aids. Genitourin Med 1991; 67: 339-341. 
9. Golger A, Ching S, Goldsmith CH, Pennie RA, Bain JR. Mortality in patients with necrotizing fasciitis. Plast Reconst Surg 2007; 119(6): 1803-1807.

10. Elke N. Fourniers gangrene: a review of 1726 cases. Brit J Surg. 2000; 87(6): 718-728.

11. Stephens BJ, Lathrop JC, Rice WT, Gruenberg JC. Fourniers gangrene: historic (1764-1978) versus contemporary(19791988). Differences in etiology and clinical importance. Amer Surg 1993; 59: 149-154.

12. Benizri E, Fabiani P, Migliori G, Chevallier D, Pevrottes A, Raucoules M, Amiel J, Mouiel J, Toubol J. Gangrene of the perineum. Urology 1996; 47: 935-939.

13. Laor E, Palmer LS, Tolia BM, Reid RE, Winter HI. Outcome prediction in patients with Fourniers gangrene. J Urol 1995; 154: 89-92.
14. Rieger UM, Gugger CY, Ferhadi J, Heider I, Andresen R, Pierer G, Scheufler O. Prognostic factors in necrotizing fasciitis and myositis: analysis of 16 consecutive cases at a single institution in Switzerland. Ann Plast Surg 2007; 58(5): 523-530.

15. Capelhuchnik P, Chia-Bin F, Klug WA. Enfermedad de Fournier. Factores que influyen sobre la mortalidad. Colo-proctology 1994; 10: 180-185.

\section{Endereço para correspondência:}

DR. WILMAR ARTUR KLUG

Alameda Ribeirão Preto, 487 - Ap. 103

CEP 01331-001 - São Paulo - SP

E-mail:klug@doctor.com 